\title{
Teaching Organic Electronics - Part II: Quick \& Easy Synthesis of the (Semi-)Conductive Polymer PEDOT: PSS in a Snap-Cap Vial
}

\author{
Amitabh Banerji ${ }^{1{ }^{1 *}}$, Stephan Kirchmeyer ${ }^{2}$, Klaus Meerholz ${ }^{2,3}$, Fabian Scharinger ${ }^{1}$ \\ ${ }^{1}$ Institute of Chemistry Education, University of Potsdam, Potsdam, Germany \\ ${ }^{2}$ COPT Center, University of Cologne, Cologne, Germany \\ ${ }^{3}$ Department of Chemistry, University of Cologne, Cologne, Germany \\ *Corresponding author: a.banerji@uni-koeln.de
}

Received January 11, 2019; Revised February 19, 2019; Accepted April 07, 2019

\begin{abstract}
Organic Electronics is an interdisciplinary and cutting-edge research field leading to innovative applications and products like ultra-thin and high-efficient organic LED displays, light-weight and transparent organic solar cells or printed organic field-effect transistors (to name only few). The core functional materials in such devices are organic (semi-)conductors like conjugated polymers, oligomers or small molecules. As a sequel to our former contribution in the World Journal of Chemical Education (Vol 6, No. 1), we present in this paper a hands-on, quick and easy experiment for the synthesis of the (semi-)conductive polymer PEDOT:PSS. This experiment can be integrated into laboratory trainings and enriches the portfolio for teachers and lab-instructors dealing with organic electronics.
\end{abstract}

\section{Keywords: PEDOT:PSS, OLED, conjugated polymers, organic semiconductors, future technology}

Cite This Article: Amitabh Banerji, Stephan Kirchmeyer, Klaus Meerholz, and Fabian Scharinger, "Teaching Organic Electronics - Part II: Quick \& Easy Synthesis of the (Semi-)Conductive Polymer PEDOT: PSS in a Snap-Cap Vial." World Journal of Chemical Education, vol. 7, no. 2 (2019): 166-171. doi: 10.12691/wjce-7-2-16.

\section{Introduction}

It is vitally important to equip today's students with scientific literacy in order for the next generation to understand technological developments that influence modern society. Smart materials are going to play a key role in tackling global problems of energy and environment (e.g. materials for energy generation and storage, low-power lighting, water splitting, carbon capture, etc.). Thus, it is necessary to ensure that cutting edge research in these area finds its way into the classroom, giving students an opportunity to perform experiments that provide hands-on experience with these materials. Organic Electronics is a modern and promising research field, leading to innovative applications like light-weight, cost-efficient, environmental-friendly, flexible or even transparent electronic modules. Since several years, our workgroup is engaged in bringing this cutting-edge research field into the classroom $[1,2,3]$. We have developed a material-kit for building low-cost Organic LED (OLED) and Organic Photovoltaic (OPV) devices based on conjugated polymers [4]. Furthermore, we have developed an experiment for the quick and easy synthesis of the semiconducting polymer MEH-PPV, which can be used in our low-cost devices $[5,6]$.

This contribution presents our latest work, the quick and easy oxidative synthesis of the (semi-) conductive polymer PEDOT:PSS, poly[(3,4-ethylenedioxy)thiophene] poly(styrenesulfonate). PEDOT:PSS is used in our OLED/OPV devices to make the processing more reliable and enhance their performance and efficiency. The following article describes the basic theoretical backgrounds on the synthesis and characterization of PEDOT:PSS along with the detailed description of the hands-on experiment for teaching purposes.

\section{Theoretical Background}

The polymer poly[(3,4-ethylenedioxy)thiophene] is commonly known as PEDOT. PEDOT belongs to the class of intrinsically conductive polymers (ICP), a class of compounds having conducting or semiconducting properties which was awarded with the Nobel prize in the year 2000 to Heeger [7], Shirakawa [8], and MacDiarmid [9]. Out of the vast number of ICP, PEDOT is recognized as the ICP most widely used in industrial applications [10].

In fact, PEDOT has helped to boost mobile phones by enabling capacitors with an extremely low ESR (equivalent series resistance) [11] to work at higher frequencies [12] and are used in the through hole plating of printed wiring boards [13], a process to connect several copper wiring layers through vias. Films of the polymeric salt poly[(3,4-ethylenedioxy)thiophene] poly(styrenesulfonate) or PEDOT:PSS films have provided electrostatic 
discharge (ESD) layers [14] and helped the display industry to increase the yield during manufacturing. In addition, PEDOT:PSS has served as hole injection layer [15] in polymer light emitting diodes (PLED) [16], as hole transport layer $[17,18,19]$ or anode [20] in organic solar cells [21,22], and as chromophore in electrochromic displays and windows [23].

In general, ICPs feature an extended $\pi$-system as the basis of their conductivity. In every-day polymers, such as the well-known polyethylene, all valence electrons form covalent $\sigma$-bonds, causing a very large energetic gap between the highest occupied molecular orbital (HOMO) and the lowest unoccupied molecular orbital (LUMO). Such materials show typically insulating properties. On the other hand, large $\pi$-systems consisting of alternating single and double bonds are formed along the polymer backbone in conjugated polymers such as PEDOT. These $\pi / \pi^{*}$-orbitals narrow the HOMO-LUMO-energy gap. As long as the $\pi$-molecular orbitals (MOs) are completely filled, pristine conjugated polymers are typically semiconductors showing only a small conductivity [24].

If, however, a conjugated polymer is "doped", very high conductivity - in some cases approaching those of metals - can be achieved. "Doping" is achieved by introducing charges into the pristine conjugated polymers, either by removing electrons from the $\pi$-MOs (oxidation) or adding electrons to the empty $\pi^{*}$-MOs (reduction). The consequence of such a redoxchemical reaction is a radical change in the electronic properties of the polymer. The preferred way to "dope" PEDOT:PSS is an oxidation process, the so-called oxidative doping, during which the PEDOT polymer chains become positively charged while PSS moieties act as counter ions.

The polymerization in this work follows the procedure originally described by Jonas et.al. [25]. The monomer 3,4-(ethylene)dioxythiophene (EDOT) is polymerized in water with HPSS present to provide counter ions and using sodium peroxodisulfate as oxidant (see Figure 1).
HPSS is used in stoichiometric excess to ensure stable dispersions. The molar ratio of thiophene groups to sulfonic acid groups resembles the ratio of in standard PEDOT:PSS dispersions in the range of $1: 1,9$ to $1: 15,2$ (corresponding to a weight ratio range of 1:2,5 up to $1: 20$ ).

Despite several approaches to fully understand the polymerization mechanism of PEDOT, just few details have been cleared up. Kinetic parameters obtained in a study carried out at Bayer AG [26] explain the polymerization as an initial slow dimer and trimer formation, a rapid chain propagation, and finally a polymerization terminating oxidation of the $\pi$ system ("oxidative doping"). In the final product, approximately one positive charge is found for every three to four thiophene rings. [27,28,29] In addition to these known reaction details several side reactions exist, such as protonation, which impact on the polymer formation. In practice, the polymerization of PEDOT:PSS proceeds smoothly at room temperature by simply mixing and stirring the reactants overnight. The polymer is formed via a paramagnetic polaron state as the first step of doping directly to the extremely stable, highly conductive and diamagnetic bipolaron state of PEDOT. The fact that both states, specifically the paramagnetic polaron, exhibit a deep blue color, has caused significant confusion in the literature. The optimal result of the polymerization is a stable, deep blue colored dispersion, which is sometimes erroneously phrased as "PEDOT-solution", however, PEDOT:PSS forms dispersions of soft, highly swollen PEDOT:PSS particles in water. Therefore, vigorous mixing during polymerization is essential to obtain a homogenous product. The distribution of dispersed PEDOT:PSS gel particles impacts the film morphology and consequently its conductivity.

After the polymerization reaction, sulfate and metal ions remain in the resulting dispersion which typically are removed afterwards from the product. These ions do not significantly impact the PEDOT:PSS properties while their removal adds a workup step to the overall reaction.
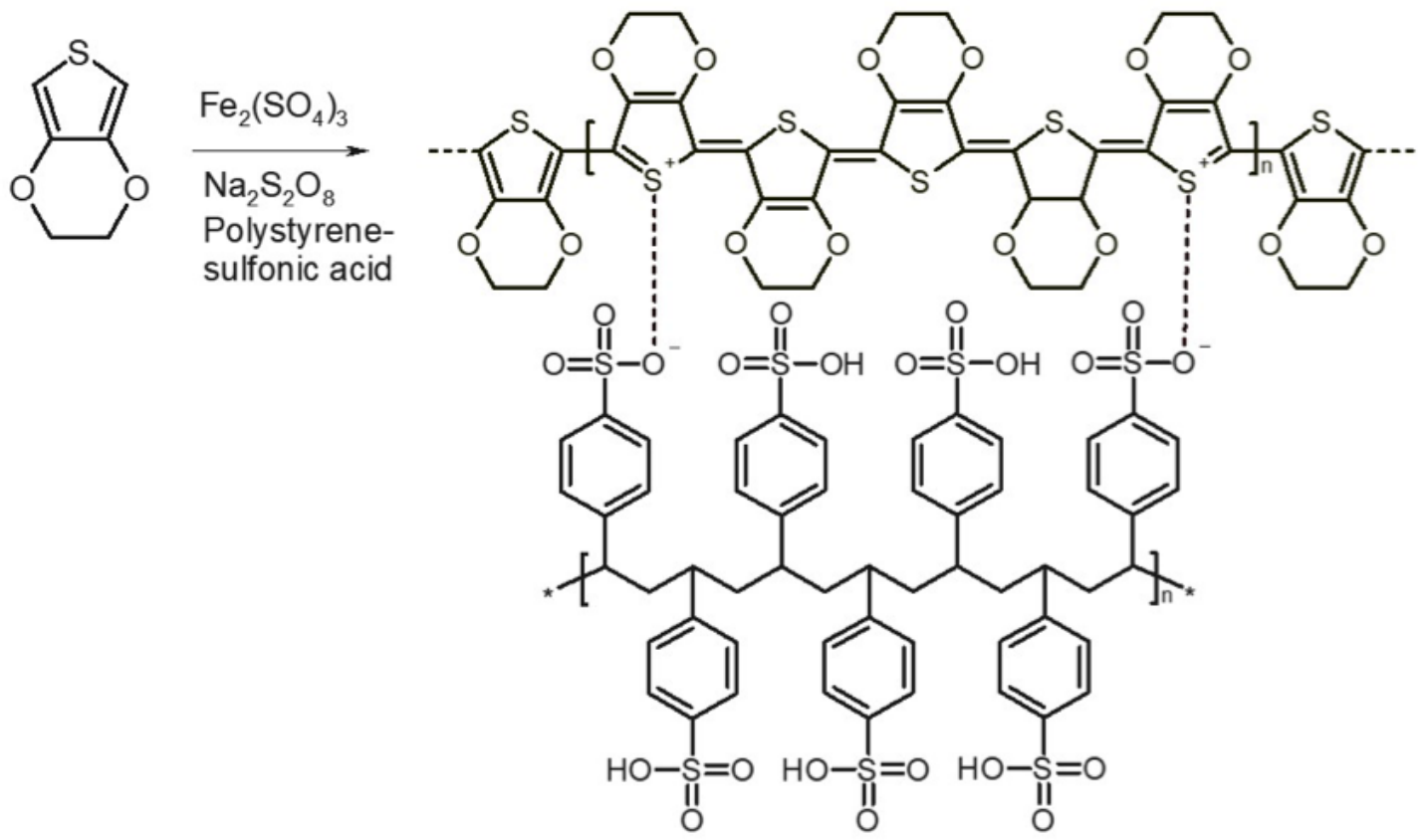

Figure 1. Polymerization Reaction Scheme of PEDOT:PSS 
Occasionally, the term "secondary dopant" is used for the addition of compounds in small quantities to PEDOT:PSS dispersions which cause a dramatic increase in conductivity. For many ICP such an increase is known and results from an increase in crystallinity of the prepared films. In the case of PEDOT:PSS a range of different explanations can be found in the literature [30]. The addition of water-miscible, high-boiling solvents like ethylene glycol or dimethyl sulfoxide to a PEDOT:PSS dispersion and heating the subsequently formed film boosts the conductivity significantly (up to a factor of 100) [31]. Often this effect can also be observed when films are dried at room temperature. However, the heating step usually helps to reach a stable and high level of conductivity [32]. Besides polar, water-miscible solvents, sugars, ionic liquids, and salts have been described as "conductivity enhancer" that remain in the film.

The conductivity of PEDOT:PSS layers is usually determined by depositing uniform thin films on to a non-conductive substrate. The sheet resistance $R_{s q}$ is measured via 4-point or 2-point probes after depositing suitable metal electrodes $[33,34,35]$. The resistivity $\rho$ or its inverse, the conductivity $\sigma$ is calculated by multiplying $\mathrm{R}_{\mathrm{sq}}$ with the layer thickness $\mathrm{d}$, according to

$$
\rho=\sigma^{-1}=R_{s q} \cdot d .
$$

The layer thickness of PEDOT:PSS films is usually determined by scanning the stylus of a high resolution mechanical profilometer across a scratch introduced to the film or by ellipsometry, without destroying the film.

\section{Experimental Part}

PEDOT:PSS can be obtained in different ways. For instance, it can be synthesized electrochemically by oxidative electro-polymerization of EDOT on a conductive glass slide (e.g. FTO or ITO glass), as described in [36,37]. We recommend the chemical synthesis by oxidative polymerization of EDOT in an aqueous solution (see chap. II), as this yields a stock of PEDOT:PSS dispersion, which can be further processed with the method of choice (e.g. spin-coating, doctorblading, printing etc.). To simplify and shorten the reaction process, we have developed a quick and easy approach, which is carried out in a small snap-cap vial and can be done within two hours.

\section{Quick \& Easy Synthesis of PEDOT:PSS in a Snap-Cap Vial}

Safety instructions: The synthesis must be conducted under a fume hood, wearing gloves, a lab coat and safety glasses. Care must be taken while working with the syringes. Materials:

1 snap-cap vial $(5 \mathrm{~mL}), 1$ small magnetic stirring rod, magnetic stirrer, 2 syringes with needles $(1 \mathrm{~mL}), 1$ measuring cylinder $(25 \mathrm{~mL})$, spatula, funnel, pipette $(3 \mathrm{~mL})$, laboratory scale

Chemicals:

- Monomer: 3,4-Ethylenedioxythiophene (T, GHS06), CAS: $126213-50-1$

- Oxidant: Potassium peroxodisulfate (O, GHS03), (Xi, GHS07), (Xn, GHS08), CAS: 7727-21-1
- Dispersant: Poly(4-styrenesulfonic acid) solution (C, GHS05), CAS: 28210-41-5

\section{Experimental Execution:}

1. Fill $2.5 \mathrm{~mL}$ of distilled water into the snap-cap vial by using the pipette. Add $0.07 \mathrm{~g}(=0.05 \mathrm{~mL})$ of the monomer and $0.09 \mathrm{~g}(=0.08 \mathrm{~mL})$ of the dispersant. Add a small stirring rod and close the snap-cap vial. Let the solution stir at a high level for about $15 \mathrm{~min}$ on the magnetic stirrer.

2. Open the snap-cap vial and add $0.135 \mathrm{~g}$ of the oxidant. Close the vial and stir the mixture for at least 2 hours at a high level on the magnetic stirrer.

Note: Figure 2 shows the color change of the solution during the steps 1 and 2 .
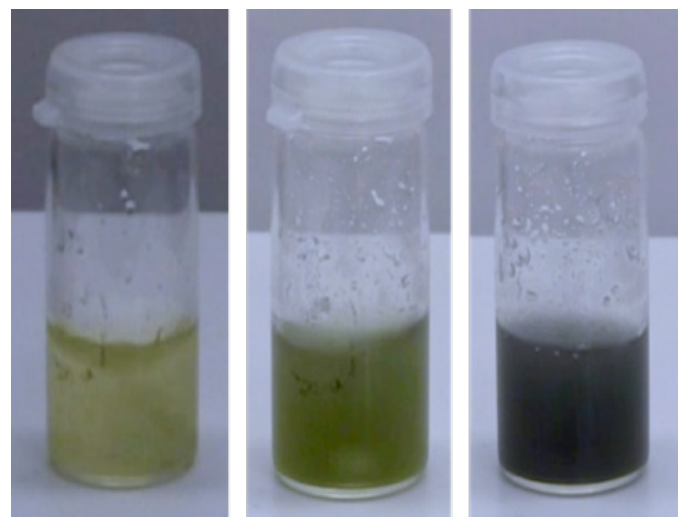

Figure 2. Observable color change during the synthesis. After $15 \mathrm{~min}$ stirring before adding the oxidant (left). One hour after adding the oxidant (centre). End of reaction after 2 hrs (right)

3. Fill $15 \mathrm{~mL}$ of distilled water into the measuring cylinder. Add the entire content of the snap-cap vial to the water (use a funnel) and stir the mixture carefully with the spatula.

4. Fill the synthesized PEDOT:PSS solution back into snap-cap vials for storage.

Note: Due the dilution with distilled water you will get a blue dispersion of PEDOT:PSS with a volume of about $17.5 \mathrm{~mL}$. Accordingly, more than three snap-cap vials can be filled with PEDOT:PSS.

Please refer to our synthesis video (QR-Code in Figure 3), where you can observe the individual steps of the PEDOT:PSS synthesis. Furthermore, you can access our step by step video-tutorials for the construction of the low-cost OLED and OPV devices.

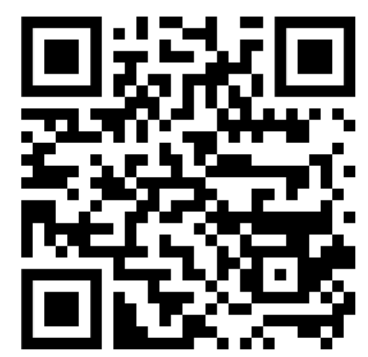

Figure 3. QR-Code to our website with video-tutorials for PEDOT:PSS synthesis and OLED/OPV device construction. (Link: www.chemiedidaktik.uni-koeln.de/oled.html)

\section{Characterization of the self-made PEDOT:PSS}

To verify the conductivity of our product, we have measured the electrical resistance of PEDOT:PSS-films, 
which we applied on a glass slide. The films were either doctor-bladed or spin-coated (at $2.500 \mathrm{rpm}$ ). To determine the sheet-resistance we used the four-point-method. As comparative trial we used commercial PEDOT:PSS from Heraeus (CLEVIOS P VP.AI 4083). All values were recorded at $\mathrm{I}=10 \mathrm{~mA}$.

Table 1. Sheet-resistance of self-made vs. commercial PEDOT:PSS films. The data have been rounded to the nearest 100-digit

\begin{tabular}{|c|c|c|}
\hline PEDOT:PSS Probe & R (Spin-coated) & R (Doctor-bladed) \\
\hline commercial & $7.700 \mathrm{M} \Omega$ & $8.600 \mathrm{M} \Omega$ \\
\hline self-made & $8.500 \mathrm{M} \Omega$ & $8.600 \mathrm{M} \Omega$ \\
\hline
\end{tabular}

The measured resistances for the PEDOT:PSS films (Table 1) show values below the resistance of pure glass, which lies between $10^{11}-10^{15} \Omega$. The spin-coated films of commercial PEDOT:PSS show lower sheet-resistances than our self-made product. For the commercial product, the doctor-bladed films have higher resistances than the spin-coated ones. Possible cause for this can lie in the missing film homogeneity caused by the microscope slides which were used as doctor-blades. These slides have rough edges, which can cause holes in the applied films.

\section{Performance of the self-made PEDOT:PSS in the OLED}

PEDOT:PSS is well known to enhance the performance and lifetime of OLED and OPV devices [38]. The scientific reason behind this is, that PEDOT is able to narrow down the energetic barrier for the hole injection (OLEDs) or extraction (OPV). Additionally, the PEDOT:PSS film smoothens the rough surface of the TCO-glass (transparent conductive oxide), which enhances the contact to the organic semiconductor layer and improves the lifetime of the devices.

We have fabricated OLED devices using a) our synthesized PEDOT:PSS, b) commercial PEDOT:PSS and c) without any PEDOT:PSS and we compared the light-densities of the devices at different voltages (Figure 4). For each sample, we have built 3 OLEDdevices with 3 emission-spots each and calculated the average light-densities. Emission-spots with a malfunction (e.g. a black spot) have been neglected in the calculation. The light-densities were measured with a GOSSEN MAVO-MONITOR USB Luxmeter.

The graphs in Figure 4 clearly show, that the lightdensities L of the OLEDs with the self-made PEDOT:PSS (orange curve) are somewhat lower than those of the devices with commercial PEDOT:PSS (blue curve), but still significantly higher than those without any PEDOT:PSS at all (grey curve). Both the curves for the devices with PEDOT:PSS show similarity in their shape and trends. This is a very satisfying validation of our "do it yourself" synthesis route for PEDOT:PSS. The graphs also reveal, that the devices using PEDOT:PSS have a lower threshold voltage and show better performance than the single-layer OLEDs as discussed before. The maximum light-densities for the devices with PEDOT:PSS lie above $100 \mathrm{Cd} / \mathrm{m}^{2}$ (at around 10-11 V) while the single-layer OLEDs reach only approx. 70 $\mathrm{Cd} / \mathrm{m}^{2}$ (at around 14-15 V). The curves decline at higher voltages because the devices start to heat up and consequently break down under loss of light-emission. To calculate the efficiency of the devices it is necessary to determine their current densities. This has been neglected at this point for simplification.

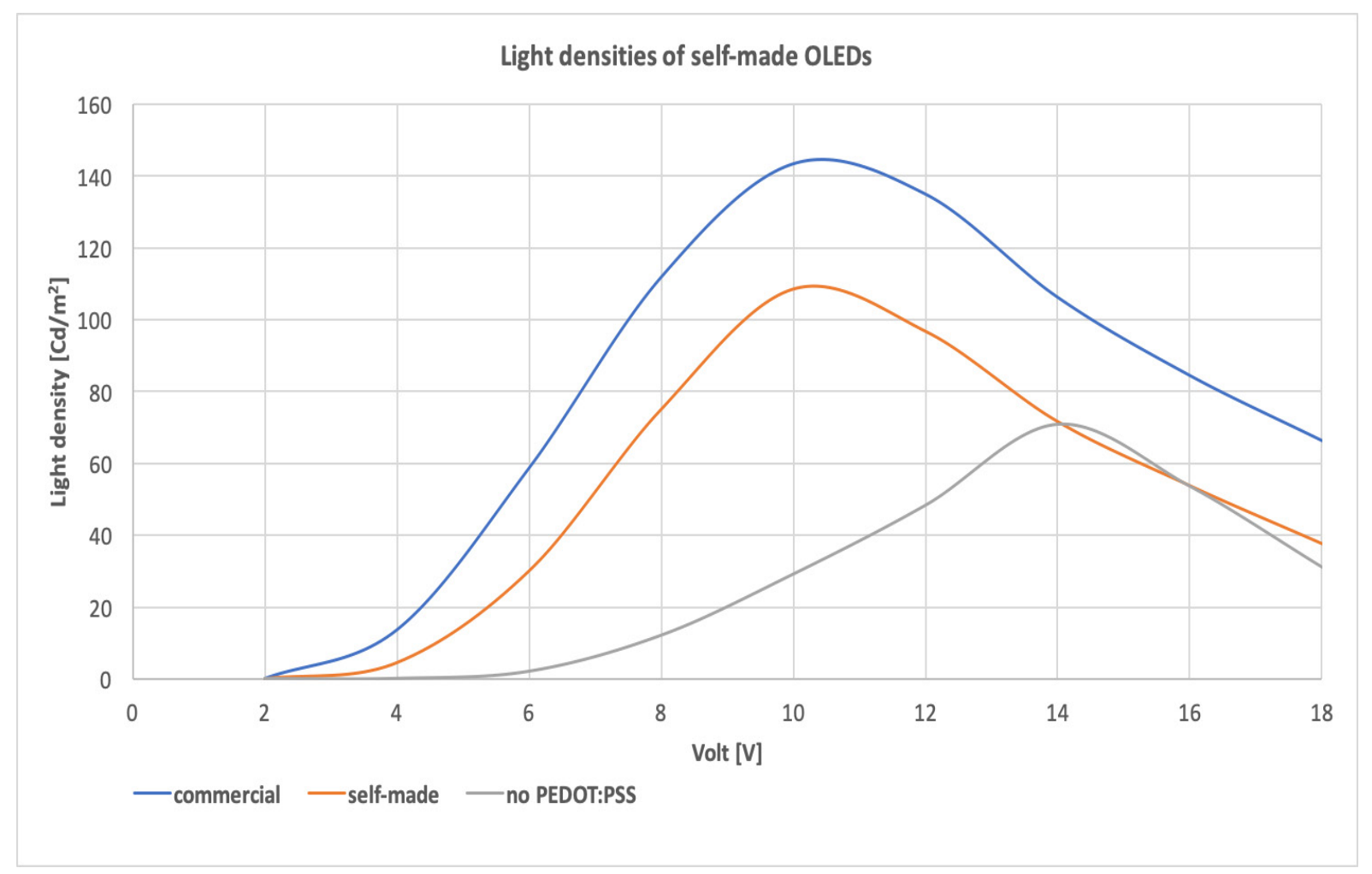

Figure 4. Light densities of OLEDs with commercial PEDOT:PSS (blue curve), OLEDs with self-made PEDOT:PSS (orange curve) and OLEDs without PEDOT:PSS (grey curve) at different voltages 


\section{Conclusion \& Outlook}

We have shown, that one does not necessarily need expensive lab equipment and a complex reaction process to synthesize the conductive polymer PEDOT:PSS. Especially for teaching purposes, it is sufficient to carry out the synthesis of PEDOT:PSS in a snap-cap vial using only small amounts of the reactants. This approach increases the safety of the experiment and decreases the reaction time and cost, which is important for laboratory trainings with limited time and resources. The characterization of our self-made PEDOT:PSS revealed a satisfying performance of the material in our OLED devices, though showing lower values in conductivity and in its performance as layer in OLED devices compared to the commercial product.

Our quick and easy synthesis of PEDOT:PSS enhances the portfolio for teaching organic electronics. It gives students an insight into the synthesis of (semi-)conductive polymers and points out the relevance of chemistry for the development and characterization of new and smart materials. The topic of organic semiconductors fits into most of the curricula of high-schools and universities. For instance, it can be applied when teaching electrochemistry or polymers. On the theoretical level, the topic allows to discuss the conjugated $\pi$-system and its influence on the conductivity of the material. Furthermore, it is possible to consider structure-property aspects, as the polyionic character of the PEDOT:PSS molecules makes the material water-soluble.

In the next research steps, we will test our experimental approach along with students to identify possible weaknesses and problems of the experiment. Also, we will try to add different "secondary dopants" to enhance the conductivity of our product as described in the theoretical part.

\section{References}

[1] Banerji, A., Tausch, M.W., Scherf, U., Classroom Experiments and Teaching Materials on OLEDs with Semiconducting Polymers. Educacion Quimica 2012, 24 (1), 17-22.

[2] Banerji, A., Tausch, M. W., Scherf, U., Fantastic Plastic - von der Cola-Flasche zur organischen Leuchtdiode. CHEMKON, 2012, 19(1), 7-12.

[3] Banerji, A., Dörschelln, J., Schwarz, D., Organische Leuchtdioden im Chemieunterricht, Chemie in unserer Zeit 2018, 52 (1), 34-41.

[4] Banerji, A., Organische Elektronik als Lehrstoff, Nachr. Chemie 2017, 65 (7-8), 807-809.

[5] Banerji, A., Schönbein, A. K., Wolff, J., OLED Reloaded: Die Synthese des Halbleiterpolymers MEH - PPV als Schulversuch, CHEMKON 2018, 24 (4), 251-256.

[6] Banerji, A., Schönbein, A.-K., Halbrügge, L., Teaching Organic Electronics: The Synthesis of the Conjugated Polymer MEH-PPV in a Hands-on Experiment for Undergraduate Students, World Journal of Chem. Educ. 2018, 6 (1), 54-62.

[7] Heeger, A. J., Semiconducting and metallic polymers: The fourth generation of polymeric materials (Nobel Lecture), a) Angew. Chem. 2001, 113(14), 2660-2682 b) Angew Chem Int Ed 2001, 40(14), 2591-2611.

[8] Shirakawa, H. The discovery of polyacetylene film: the dawning of an era of conducting polymers (Nobel Lecture), a) Angew Chem 2001, 113(14), 2642-2648 b) Angew Chem Int Ed 40(14), 2575-2580.

[9] MacDiarmid, A. G. , "Synthetic Metals": A novel role for organic polymers (Nobel Lecture). a) Angew Chem 113(14), 2649-2659 b) Angew Chem Int Ed 2001, 40(14), 2581-2590.
[10] Elschner, A., Kirchmeyer, S., Lövenich, W., Merker, U., Reuter, K., PEDOT: Principles and Applications of an Intrinsically Conductive Polymer, 2010, CRC Press, Boca Raton.

[11] Merker, U., Wussow, K., Kirchmeyer, S., Schnitter, C. and Lerch, K.. Manufacturing Process for Low ESR Polymer Electrolyte Capacitors. Proceedings of the $17^{\text {th }}$ Passive Components Conference CARTS Europe, 2003, 79, Stuttgart.

[12] Reed, E., Marshall, J. and Hahn, R., How low can you go tantalum polymer capacitors with ESR under $7 \mathrm{~m} \Omega$. In: Proceedings of the 16th passive components symposium CARTS Europe, 2002, 60-67, Electronic Components Institute Internationale Ltd., Port St. Laurent.

[13] Jonas, F. and Wolf, G.-D.. EP 553671 (Bayer AG). Prior: 29 January 1992

[14] Frisch, K. C. and Patsis, A., Triboelectrification of Polymers. In: Electrical Properties of Polymers, Ed. D. A. Seanor. 1982, 37-58. Academic Press, New York.

[15] Jonda, C., Mayer, A. B. R., Stolz, U., Elschner, A. and Karbach, A., Surface roughness effects and their influence on the degradation of organic light emitting devices, J Mater Sci 2000, 35, 5645-5651.

[16] Burroughes, J. H., Bradley, D. D. C., Brown, A. R., Marks, R. N., Mackey, K., Friend, R. H., Burns, P. L. and Holmes, A. B., Light-emitting diodes based on conjugated polymers. Nature 1990 $347,539-541$.

[17] Aernouts, T., Geens, W., Poortmans, J.. Heremans, P., Borghs, S. and Mertens, R., Extraction of bulk and contact components of the series resistance in organic bulk donor-acceptor-heterojunctions", Thin Solid Films 2002, 403, 297-301.

[18] Zhang, F. L., Gadisa, A., Inganaes, O., Svensson, M. and Andersson, M. R., Influence of buffer layers on the performance of polymer solar cells. Appl. Phys. Lett 2004, 84, 3906-3908.

[19] Yoo, I., Lee, M., Lee, C., Kim, D. W., Moon, I. S. and Hwang, D. H., The effect of a buffer layer on the photovoltaic properties of solar cells with P3OT:fullerene composites. Synth. Met. 2005, 153, 97-100.

[20] Arias, A. C., Granström, M., Thomas, D. S., Petritsch, K. and Friend, R. H. Doped conducting-polymer-semiconductingpolymer interfaces: Their use in organic photovoltaic devices. Phys. Rev. B 1999, 60(3), 1854-1860.

[21] Organic Photovoltaics: Materials, Device Physics, and manufacturing Technologies, ed. Brabec, C., Dyakonov, V. and Scherf, U., 2008, Wiley-VCH GmbH \& Co. KGaA, Weinheim.

[22] Shaheen, S. E., Ginley, D. S. and Jabbour, G. E., Organic-Based Photovoltaics: Toward Low-Cost Power Generation. MRS Bull. 2005, 30, 10-19.

[23] Heuer, H. W., Wehrmann, R. and Kirchmeyer, S., Electrochromic Window Based on Conducting Poly(3,4-ethylenedioxythiophene)Poly(styrene sulfonate). Adv. Funct. Mater. 2002, 12(2), 89-94.

[24] Heeger, A. J. Semiconducting and Metallic Polymers: The Fourth Generation of Polymeric Materials. J. Phys. Chem. B. 2001, 105(36), 8475-8491.

[25] Jonas, F. and Krafft, W., EP 440957 (Bayer AG), Prior: 20 December 1990

[26] a) Tracht, U., Bayer AG, Leverkusen, Personal Communication 2001, b) Kirchmeyer, S., Reuter, K. and Simpson, J., Poly(3,4Ethylenedioxythiophene) - Scientific Importance, Remarkable Properties, and Applications. In: Handbook of Conducting Polymers $3^{\text {rd }}$ ed. Ed. T. A. Skotheim and J. A. Reynolds, 10-1-1022. 2007, CRC Press Boca Raton.

[27] Zotti, G., Zecchin, S., Schiavon, G., Louwet, F., Groenendaal, L., Crispin, X., Osikowicz, W., Salaneck, W. and Fahlman, M., Macromolecules 2003, 36, 3337-3344.

[28] Aasmundtveit, K. E., Samuelsen, E. J., Pettersson, L. A. A., Inganäs, O., Johansson, T. and Feidenhans'l, R., Structure of thin films of poly(3,4-ethylene dioxythiophene). Synth. Met. 1999, 101(1-3), 561-564.

[29] Hwang, J., Tanner, D. B., Schwendeman, I. and Reynolds, J. R.. Optical properties of nondegenerate ground-state polymers: Three dioxythiophene-based conjugated polymers Phys. Rev. B 2003, 67(11), 115205-1-115205-10.

[30] Ouyang, J., Xu, Q., Chu, C.-W., Yang, Y., Li, G. and Shinar, J., On the mechanism of conductivity enhancement in poly $(3,4-$ ethylenedioxythiophene):poly(styrene sulfonate) film through solvent treatment. Polymer 2004, 45 (25), 8443-8450.

[31] Jonas, F., Karbach, A., Muys, B., van Thillo, E., Wehrmann, R., Elschner, A. and Dujardin, R., EP 686662 (Bayer AG), Prior: 6 May 1994. 
[32] Jönsson, S. K. M., Birgerson, J., Crispin, X., Greczynski, G., Osikowicz, W., Denier van der Gon, A. W., Salaneck, W. R. and Fahlman, M., The effects of solvents on the morphology and sheet resistance in poly(3,4-ethylenedioxythiophene)-polystyrenesulfonic acid (PEDOT-PSS) films. Synth. Met. 2003, 139(1), 1-10.

[33] Giraudet, L., Fauveaux, S., Simonetti, O., Petit, C., Blary, K., Maurel, T., and Belkhir, A., Spin-coated conductive polymer film resistivity measurement using the TLM method. Synth. Met. 2006, 156(11-13), 838-884.

[34] Bantikassegn, W. and Inganäs, O., Electronic properties of junctions between aluminum and doped poly(3,4-ethylenedioxythiophene). Thin Solid Films 1997, 293(1-2), 138-143.
[35] Jönsson, S. K. M., Salaneck, W. R. and Fahlman, M., X-ray photoelectron spectroscopy study of the metal/polymer contacts involving aluminium and poly(3,4-ethylenedioxythiophene)poly(styrenesulfonic acid) derivatives. J. Mater. Res. 2003, 18(5), 1219-1226.

[36] Scherr, D., Plastiksolarzellen-ein Experiment für die Schule?. CHEMKON 2014, 21(1), 31-36.

[37] Rendon-Enriquez, I. N., Tausch, M. W., Scherf U., Elektrochrome Fenster mit leitenden Polymeren.Chem. unserer Zeit 2016, 50, 400-405.

[38] Hertel, D. Müller, C.D., Meerholz, K. Organische Leuchtdioden: Bilderzeugung, ChiuZ 2005, 39 (5), 336-347.

(C) The Author(s) 2019. This article is an open access article distributed under the terms and conditions of the Creative Commons Attribution (CC BY) license (http://creativecommons.org/licenses/by/4.0/). 